\title{
Wide Band Frequency Agility Direct Frequency Synthesizer and Its Performance Analysis
}

\author{
Huang Shizhao \\ Newstar Information Technology Inc. \\ Hefei, China \\ e-mail: huang_sz@163.com \\ Yang Yulin \\ Newstar Information Technology Inc. \\ Hefei, China
}

\author{
Wang Liujun \\ Newstar Information Technology Inc. \\ Hefei, China \\ Wang Xin \\ Newstar Information Technology Inc. \\ Hefei, China
}

\begin{abstract}
This paper will introduce the design of wideband frequency agility synthesizer based on method of Simulate direct frequency synthesis, the full-phase coherence Modulation and Filter bandwidth control are applied in this synthesizer as designed .The main factors and solutions of the effect of the frequency switching time are analyzed and the frequency change time of the system is tested. The result shows that the frequency switching time between any two frequencies is less than $0.8 \mu$ s on the full band.
\end{abstract}

Keywords-Frequency Agility; Direct synthesis; Frequency switching time

The typical characteristics of the radar signal are wide band, frequency agility, wave complexity and diversity, simulate direct frequency synthesizer must possess large bandwidth, small step and frequency Agility and can inhibit the clutter within band for achieving realistic simulation of battlefield radar signal environment, so it increases high requirements for the design of frequency synthesizer. Now, the design of frequency synthesizer generally uses phase locked and direct synthesis, when working in the wide band, the defect of the phase locked will seriously deteriorate the output of phase noise, make frequency switching time more slow and can't simulate the advanced radar signals of less frequency switching time. Compared with it, the full-phase direct frequency synthesizer have better frequency stability and less frequency hopping time to meet the need of radar signal environment simulation.

Therefore, in order to achieve high quality radar signals, the real-time building complex radar signal environment selects relatively complex technology, but the simulation direct frequency synthesis methods of high performance index to develop the required frequency source of systems. And comprehensively uses of modular hierarchical design methods in the design, high performance filter, and effectively control the signal spurious level, decreases the volume of the system.

\section{SYSTEM STRUCTURE}

System design for signal range is $2 \sim 18 \mathrm{GHz}$, as high as 9 octave, stepped frequency is $10 \mathrm{MHz}$, in order to ensure the quality of the output signal, we must avoid low harmonic and low intermodulation of the signal. The frequency domain must be sub-band when conduct. At the same time, we must attend to the quantity of equipment can not be too large, in harmonic suppression and clutter suppression index and in meet the normal working condition, the $2 \sim 18 \mathrm{GHz}$ bandwidth of frequency division modulation (FDM) will be channelized and divided into four band transmission channels:2 4GHz, $4 \sim 8 \mathrm{GHz}$, $8 \sim 12 \mathrm{GHz}, 12 \sim 18 \mathrm{GHz}$. Output four microwave signals of different carrier frequencies and different wave forms, carrier frequency step is $10 \mathrm{MHz}$, the signals generated by the frequency source subsystem should be self coherent, all signals are fully coherent.

(1) Circuit structure

Principle diagram as shown in Figure1, Wide band frequency source is divided into three parts, the first part through direct frequency synthesis way segmented produces $\mathrm{L}$ band, $\mathrm{S}$ band and $\mathrm{X}$ band of the local oscillator signals, respectively, as the local oscillator of the 1 , the 2 and the 3 . Firstly, the $\mathrm{L}$ band of the local oscillator signal is generated, becomes the two outputs, one to produce $\mathrm{X}$ band of the local oscillator signal, the other is sent to a transmission channel 4, producing $12 \sim 18 \mathrm{GHz}$ transmitting signal. Using $\mathrm{L}$ band local oscillator1 generates a $\mathrm{X}$ band of the local oscillator signal, then generates $\mathrm{S}$ band of the local oscillator signal, the three local oscillators are not independent of each other, have a certain relationship. Through the local oscillator signal the second part move the waveform signal to the desired transmission frequency band, and amplify it to the appropriate band. Each band has a transmitting channel, are known as the transmitting channel $\mathrm{S}$, the transmitting channel $\mathrm{C}$, the transmitting channel $\mathrm{X}$ and the transmitting channel KU. The third part is the waveform signal generator, producing various forms of radar signals. 


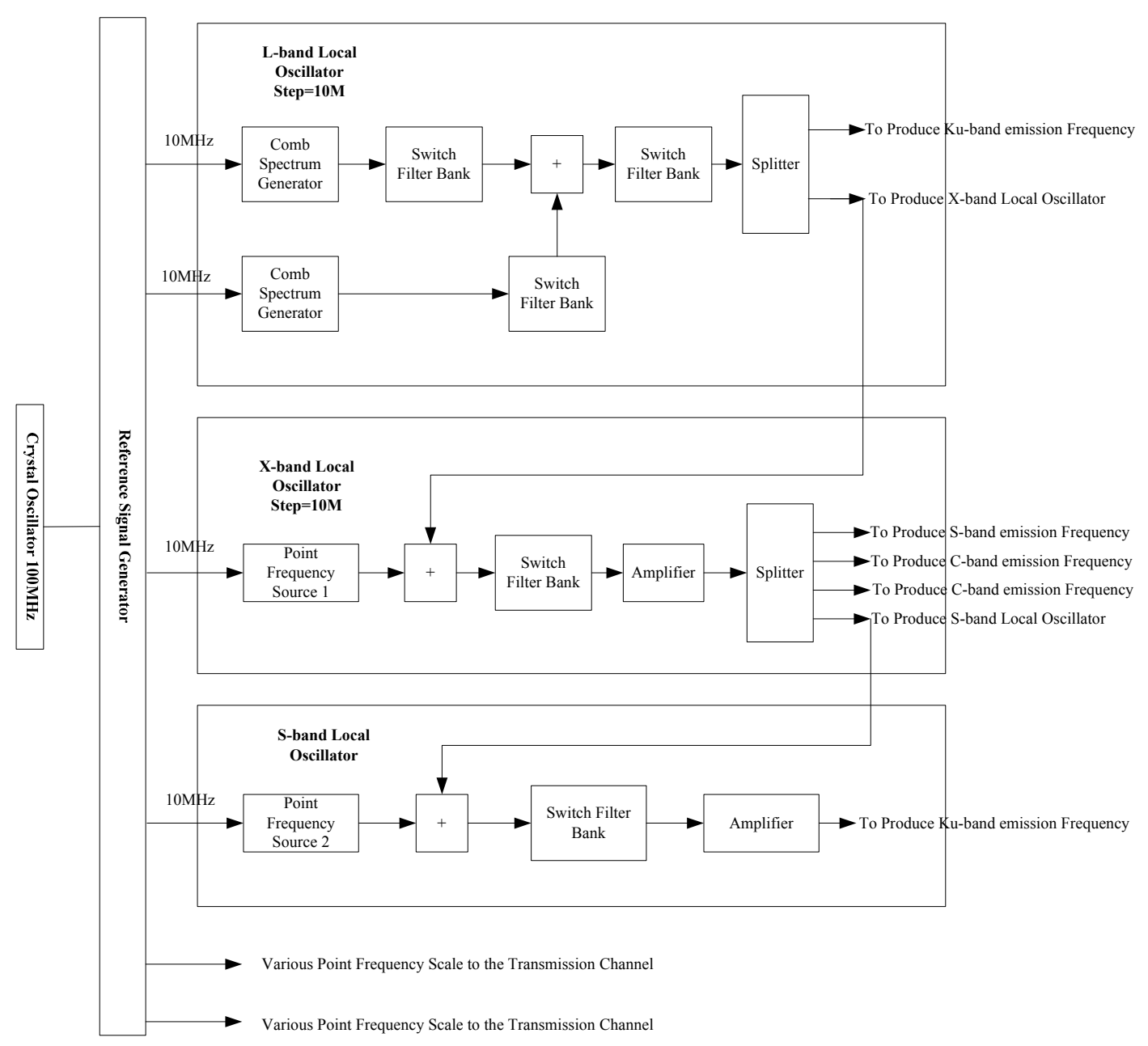

Figure 1 Wide Band Frequency Agility Direct Frequency Synthesizer Principle Diagram

(2)Signal transformation relationship

Firstly, selecting $100 \mathrm{MHz}$ oscillator signal, then the reference signal generator generates a $10 \mathrm{MHz}$ signal, the comb spectrum generator generates a set of spaced $10 \mathrm{MHz} \mathrm{P}$ band signals, at last, and selects a $\mathrm{P}$ band frequency standard signal through the switching filter group. The reference signal generator generates a $200 \mathrm{MHz}$ signal, the comb spectrum generator generates a set of spaced $200 \mathrm{MHz} \mathrm{L}$ band signals, and selects an L band frequency standard signal through the switching filter group. The $\mathrm{P}$ band frequency standard signal and the $\mathrm{L}$ band frequency standard signal mixing produces al $2 \mathrm{GHz}$ microwave signal. The $\mathrm{L}$ band local oscillator signal output is divided into two, one is sent to the $\mathrm{X}$ band transmitting channel to produce $8 \sim 12 \mathrm{GHz}$ emission signals, the other to the $\mathrm{X}$ band local oscillator source, for generating a $10 \sim 12 \mathrm{GHz} \mathrm{X}$ band local oscillator signal.

The $\mathrm{L}$ band local oscillator signal and the $\mathrm{X}$ band frequency standard signal mixing produces a $10 \sim 12 \mathrm{GHz}$ local oscillator signal, because the resulting signal is wide-band signal, mixing link will produce rich combined frequency, the switching filter extracts the useful signal, filtering out useless signals, in order to ensure the signal quality excellent .The $\mathrm{X}$ band local oscillator signal is divided into four outputs, one for generating a $2 \sim 4 \mathrm{GHz} \mathrm{S}$ band local oscillator signal, the rest to the transmitting channel to generate the transmitting signal. These three band local oscillator signals performance largely determine the performance of the whole system.

\section{FREQUENCY CONVERSION TIME AND CONTROL METHOD}

Microwave frequency synthesizer switching speed depends mainly on the microwave PIN switch's working speed and switching time of driving circuit's switch. Switching speed is applied to the output amplitude and frequency, which is the required for the delay time of synthesizer changing from one frequency to another frequency or from a power level to another power level or frequency and power level changing at the same time. The delay time is a sum of the synthesizer processor command response time, switching / blanking time, residence time, setting time and other factors. Switching / blanking time is the delay time that the new frequency achieve stability 
from the parallel BCD codes sent to the synthesizer to the final phase of the output reaching 0.1 radians or within the given new frequency tolerance. In order to avoid appearing harmonic or false signal at output port when switching, blanking means closing the synthesizer RF power output when transmitting one frequency to another frequency.

From the above analysis, in order to reduce the system frequency conversion time, basically took the following steps:

(1) Using high speed processor and digital logic devices, reducing the response time of the processor

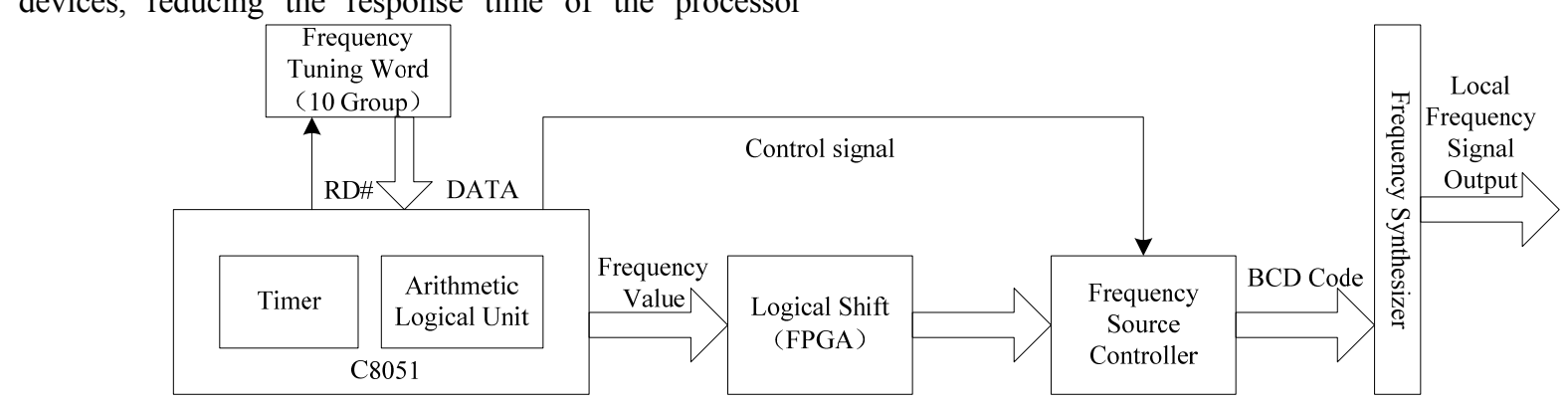

Figure 2 Frequency switching control structure diagram command.

The output frequency of the frequency synthesizer system is controlled by the main control system processor, when in a frequency agile state, the main control MCU will provide a channel maximum 10 frequency points, the control computer timed reading frequency value from memory in the timer control, and after the logical conversion this value will be sent to the synthesizer controller, then the frequency synthesizer outputs corresponding the frequency signal by the controller, the control structure as shown in figure 2 .
Figure 2 shows: the processor command response speed depends mainly on the processor's operation speed, the bus speed, the FPGA operation delay, the data input / output delay of frequency synthesizer controller. And the basic method of reducing time is to improve the speed of processor and digital logic device.

a. The system processor uses the improved type 8051 micro controller of frequency $22 \mathrm{MHz}$ that the bus cycle is $45 \mathrm{nS}$, the ALTERA company EP1K100 high speed FPGA special device that the highest gate rate is about $250 \mathrm{MHz}$ and the corresponding gate delay is about $4 \mathrm{~ns}$. Memory uses high-speed SRAM that eliminates the delay time of MCU data reading and writing, at the same time, all use the FPGA hard-wired logic implementation to interpret the most complicated command words for operation, its speed is 100 times faster than the general MCU processor, so the time delay of this part's operation caused can be neglected. The above factors into account, the total processor command response time is not more than 100 ns.

b. Using high speed microwave device to control the frequency switching / blanking time

The switching rate of frequency synthesizer depends on the needed time from frequency controller transmitting the $\mathrm{BCD}$ code to implementing device, and effectively reduces the time of the $\mathrm{BCD}$ code transmitting and execution by using high speed microwave devices when designed.

c. Strictly controlling the bandwidth of the filter.

Generally, the new frequency setting time and filter bandwidth have a relationship, and all synthesizers use a filter, either active or passive. In many cases, the narrowest bandwidth of the filter will affect the system switching time. According to experience, the basic relationship of the general filters' $3 \mathrm{~dB}$ bandwidth and the rise time is $t_{r}=k / \Delta B_{3 d b}$, in which $0.3 \leq k \leq 0.45$, and $t_{r}$ is the required time of the pulse rising from $10 \%$ to $90 \%$.

The above factors, the total frequency conversion time is about $0.8 \mu \mathrm{s}$, after the measurement, the system frequency conversion time is $0.8 \mu \mathrm{s}$, consistent with the theoretical analysis results.

\section{TEST AND RESULTS}

Using a X band superheterodyne receiver to produce a more precise frequency agile time test circuit, joining the driving control signal in the input of the control module, controlling the output frequency the frequency synthesizer according to the rule change, also can make the frequency synthesizer jump from an arbitrary frequency to another frequency, simulate the control signal of the radar console to be frequency agility, then we borrow a hypervelocity detector to output detection signals at the frequency synthesizer output port, and can directly read the frequency agile time ( already contains the detection output delay of HP pulse power meter ) from the oscilloscope in contrast with the synchronous clock edge, such as test circuit as shown in figure 3 . 


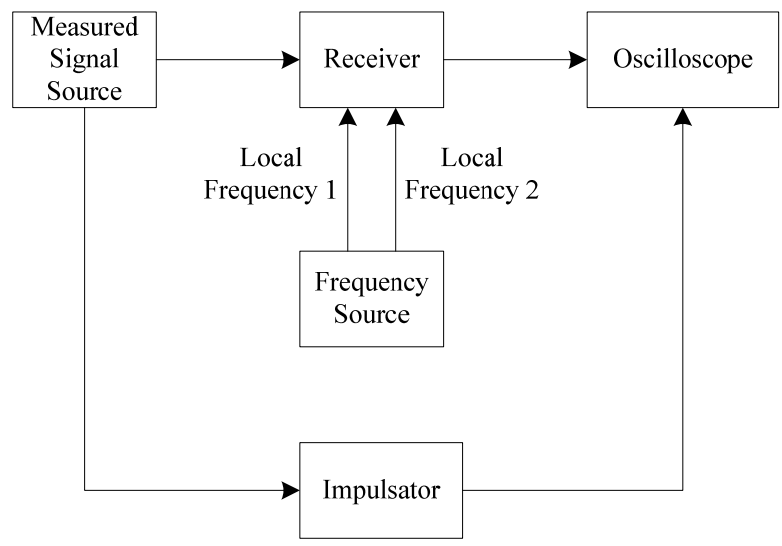

Figure3 Principle diagram of frequency switching speed test

When the frequency source working at $9.5 \mathrm{GHz}$, the difference of the super heterodyne receiver and the frequency source frequency is $60 \mathrm{MHz}$, then the test results as shown in figure 4. By the graph 4visible, at the typical frequency points, the system frequency conversion time is $800 \mathrm{~ns}$.

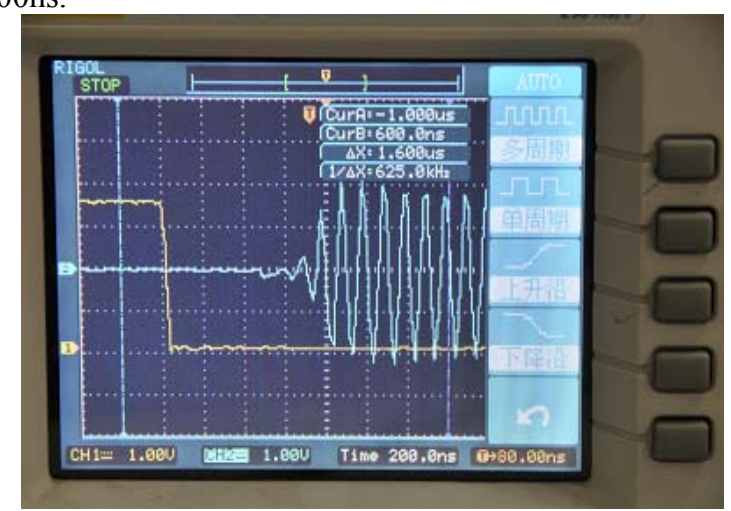

Figure4 The display frequency switching time of the oscilloscope

\section{THE END}

With phase locked frequency synthesis methods, direct frequency synthesizer frequency has fast conversion speed, high precision, low phase noise. Direct frequency synthesis technique, in order to achieve system design for frequency source part of the large bandwidth, small step, high stability requirements, and the system frequency source uses the simulation direct frequency synthesis technology, and its frequency conversion speed is faster than that of PLL technology. Using the simulation direct frequency synthesis technology, the system realized signal wideband small step output, the use of high performance filter solved the signal of large spurious and other issues, so that the signal in the whole frequency band can keep high quality.

\section{REFERENCE}

[1] Wei sheng Liu. The wideband high stability, design and analysis of low phase noise direct frequency synthesizer [J]. Journal of microwaves, 2006, 22(4): 57-61.

[2] Xiaohua Yun, Cai hua Yun, and so on. The frequency synthesizer for subminiature high speed acquisition receiver [J]. Journal of Nanjing University of Science and Technology, 1995,19(2) : 125- 127

[3] Sihong Wang. A L band broadband fast frequency synthesizer [J]. Vehicle control technology,1998,17(3) : 66-70

[4] Zai WangDong, Yaqin Chen, Youhua Lei, and so on. Communication circuit theory (Second Edition) [M]. Beijing: Higher Education Press, 2002, 503-504. 